Revue des patrimoines

\title{
Les immeubles numéros 15, 17, 19, 21 et 23 de la rue Gasparin à Lyon, un exemple urbain de production complexe du bâti
}

\section{Bernard Gauthiez}

\section{(2) OpenEdition}

Journals

Édition électronique

URL : http://journals.openedition.org/insitu/1181

DOI : 10.4000/insitu. 1181

ISSN : 1630-7305

Éditeur

Ministère de la Culture

\section{Référence électronique}

Bernard Gauthiez, «Les immeubles numéros 15, 17, 19, 21 et 23 de la rue Gasparin à Lyon, un exemple urbain de production complexe du bâti », In Situ [En ligne], 2 | 2002, mis en ligne le 24 janvier 2012, consulté le 15 novembre 2019. URL : http://journals.openedition.org/insitu/1181 ; DOI : 10.4000/insitu. 1181

Ce document a été généré automatiquement le 15 novembre 2019.

\section{(c) $(1)$}

In Situ Revues des patrimoines est mis à disposition selon les termes de la licence Creative Commons Attribution - Pas d'Utilisation Commerciale - Pas de Modification 4.0 International. 


\title{
Les immeubles numéros $15,17,19$, 21 et 23 de la rue Gasparin à Lyon, un exemple urbain de production complexe du bâti
}

\author{
Bernard Gauthiez
}

1 Une monographie d'architecture se construit d'abord par l'identification de l'édifice qui sera ensuite étudié. Cette identification est en général aisée, mais, du fait de l'histoire complexe du lieu ou de conditions de production particulières, cette opération peut s'avérer difficile, voire impossible. Tel est le cas présenté ici.

\section{L'architecture des numéros 15 à 23, rue Gasparin, à Lyon}

2 Les nos 15 à 23 de la rue Gasparin forment une série d'immeubles aux caractéristiques individuelles au premier abord peu remarquables, à la fois dans leur environnement construit et dans la production moyenne de la ville à l'époque de leur édification. 
Figure 1

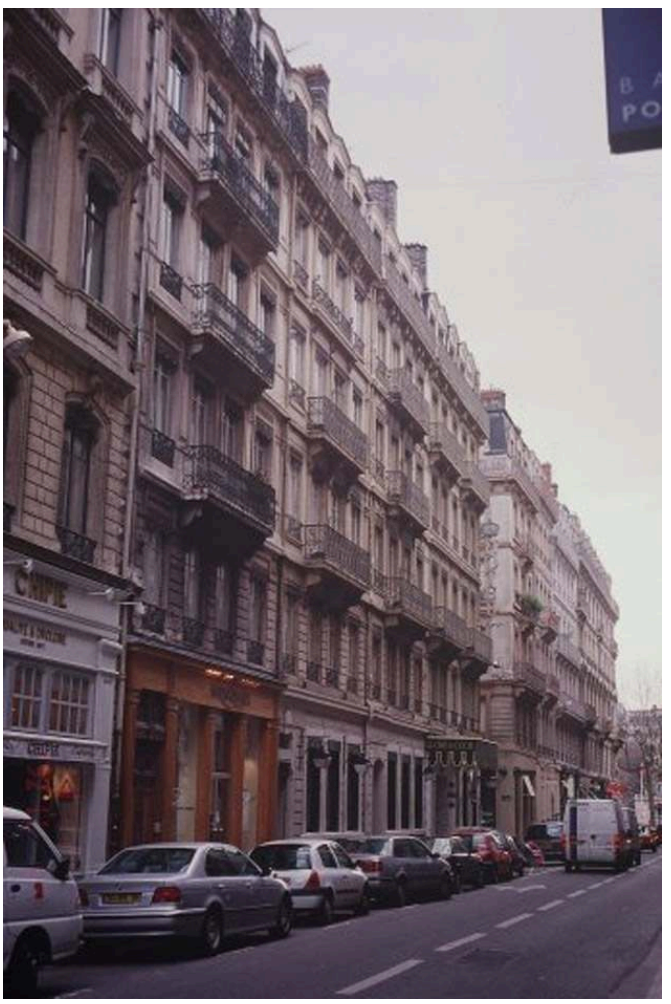

Vue d'ensemble des immeubles nos 15 à 23 rue Gasparin à Lyon, depuis le nord-ouest Phot. B. Gauthiez. (c) B. Gauthiez. 2000

3 Toutefois, un examen attentif montre qu'ils ont en commun un grand nombre de traits architecturaux, mais pas tous. Leur hauteur est identique, de même la hauteur de leurs niveaux, la forme des baies, le dessin et le détail des lucarnes. 
Figure 2

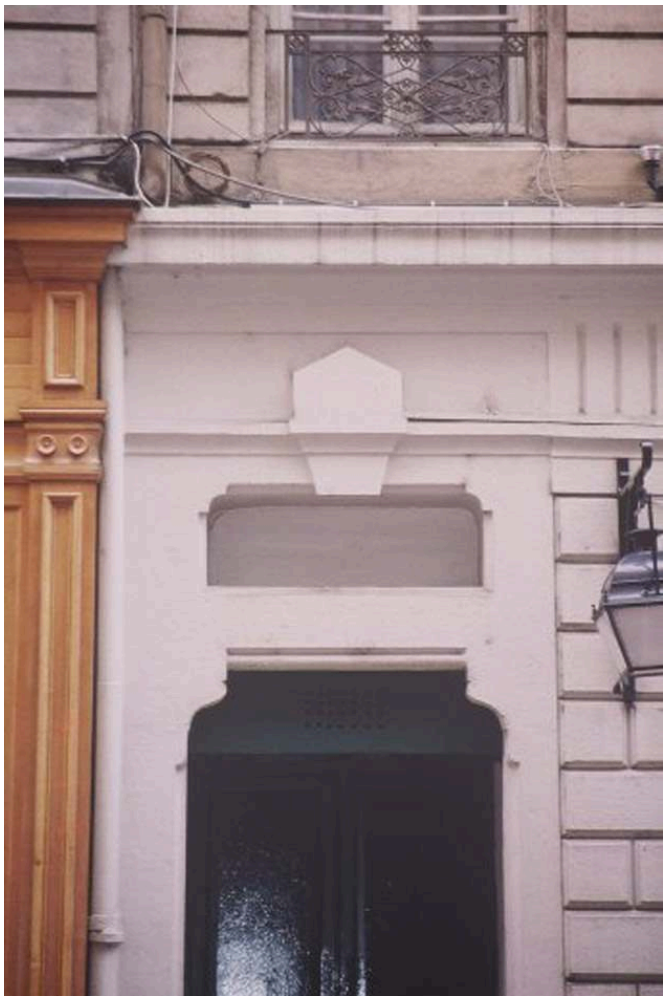

Décor de la porte d'entrée du n 17, rue Gasparin

Phot. B. Gauthiez. (c) B. Gauthiez. 2000

4 En revanche, ils se différencient par le décor secondaire, celui des portes d'entrée, par exemple celles du $n^{\circ} 17$ et du $n^{\circ} 19$, des corbeaux supports des consoles de balcons, des garde-corps, ou encore des trumeaux du premier étage. 
Figure 3

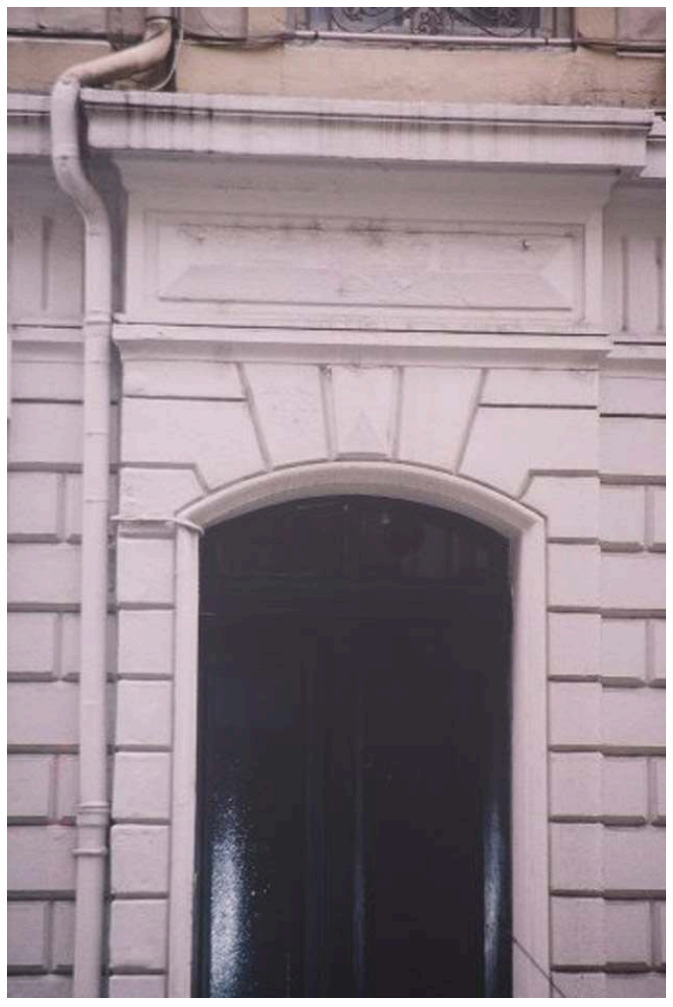

Décor de la porte d'entrée du n 19, rue Gasparin

Phot. B. Gauthiez. (c) B. Gauthiez. 2000 
Figure 4

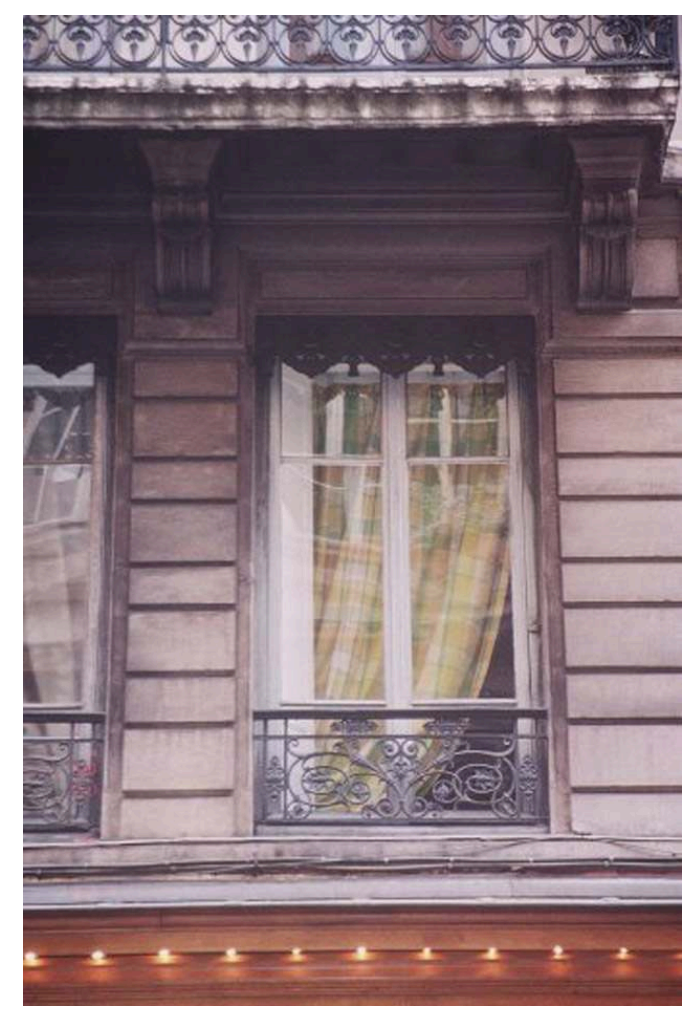

Décor de la baie du n 15, rue Gasparin

Phot. B. Gauthiez. (c) B. Gauthiez. 2000 
Figure 5

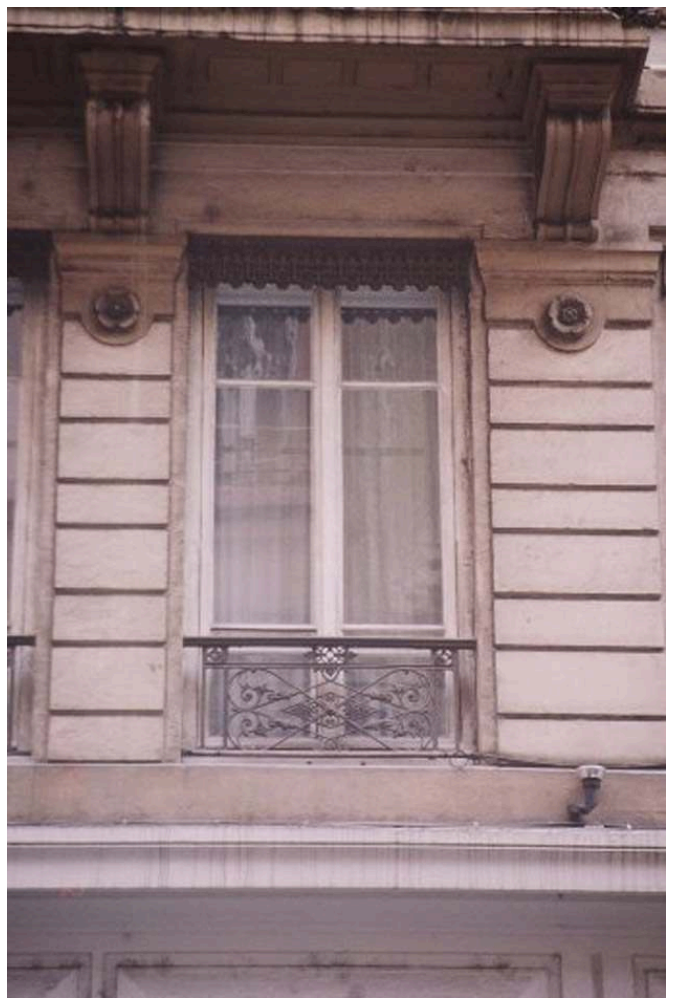

Décor de la baie du n 17, rue Gasparin

Phot. B. Gauthiez. (c) B. Gauthiez. 2000

5 Ces différences sont bien illustrées par les baies d'étage du $n^{\circ} 15$ et du $n^{\circ} 17$, placées dans des compositions de façade voisines, aux subtiles variations : entre les $\mathrm{n}^{\circ} 17$ et 19 , ou entre les nos 19 et 21. 
Figure 6

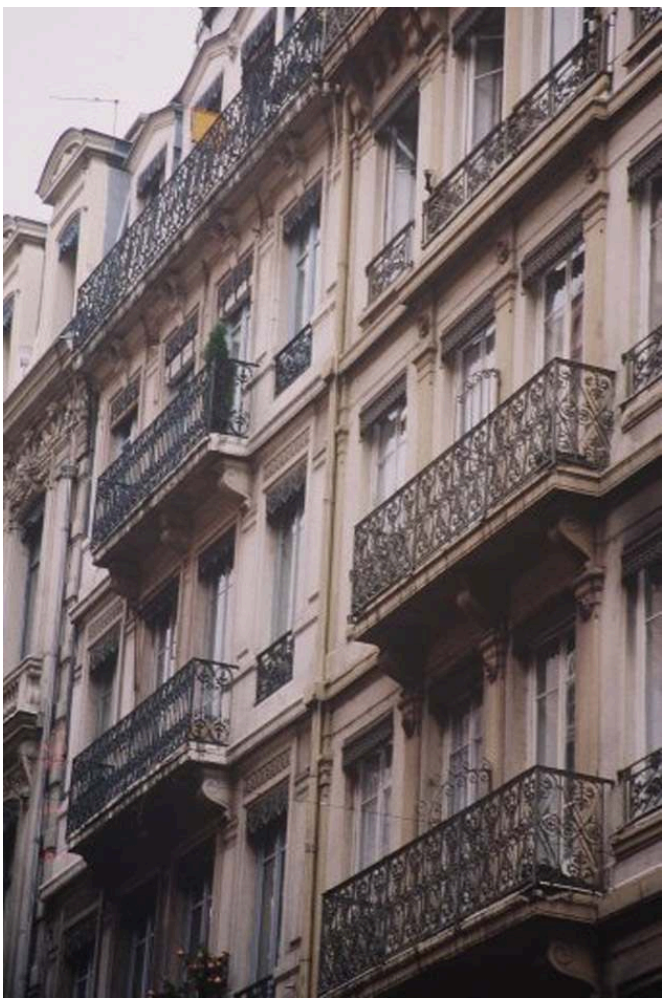

Vue des façades des nos 15 et 17, rue Gasparin, montrant à la fois la similitude du traitement architectural d'ensemble et la différence des décors secondaires

Phot. B. Gauthiez. (c) B. Gauthiez. 2000 
Figure 7

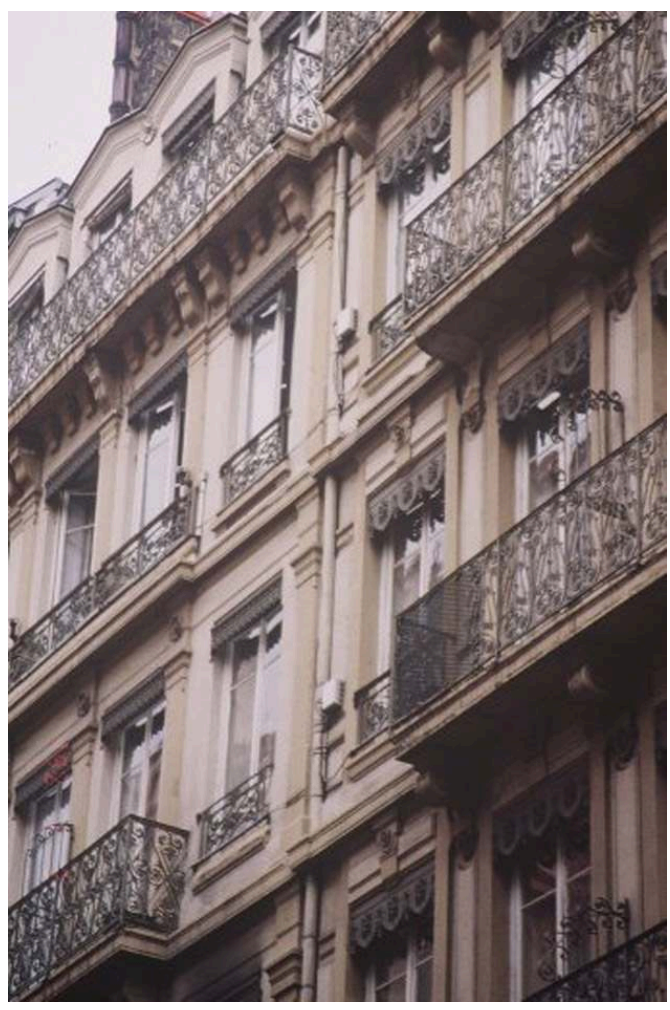

Vue des façades des nos 17 et 19, rue Gasparin

Phot. B. Gauthiez. (c) B. Gauthiez. 2000 
Figure 8

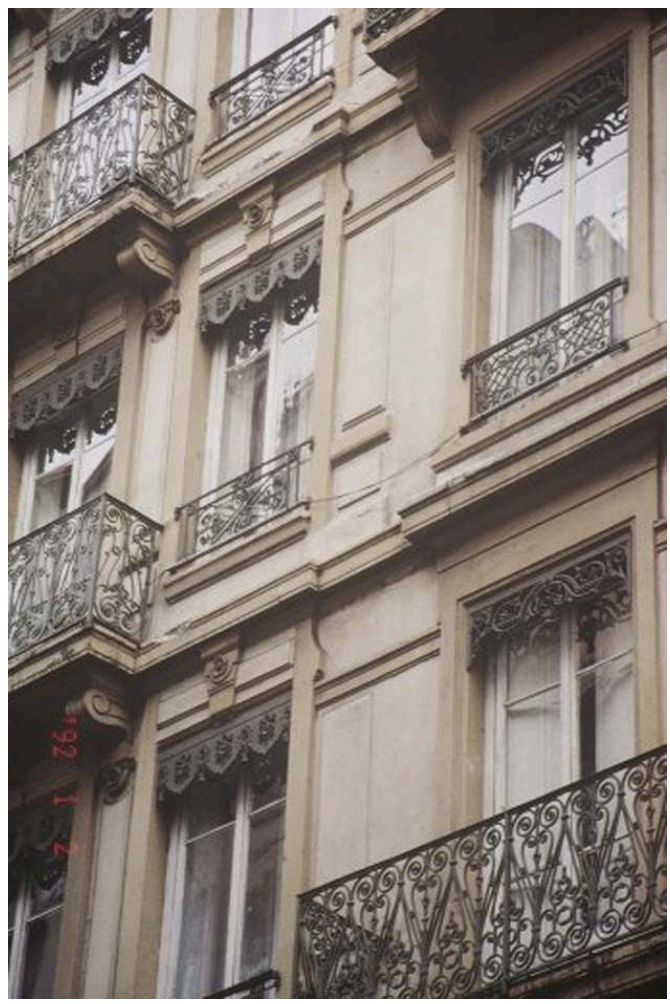

Vue des façades des nos 19 et 21, rue Gasparin

Phot. B. Gauthiez. (c) B. Gauthiez. 2000

6 Dans l'ensemble, donc, leurs caractéristiques morphologiques sont quasiment identiques, mais ils diffèrent dans le détail. Les distributions intérieures sont elles aussi les mêmes, un couloir desservi par la porte d'entrée mène à un escalier qui donne accès à chaque étage à un seul appartement. Il s'agit de petits immeubles construits sur d'étroites parcelles, destinés à la location, à quatre étages carrés et un étage de comble, comportant un faible nombre de logements (5) et un local d'activité au rez-de-chaussée.

7 Ces immeubles ne sont pas seulement morphologiquement très proches les uns des autres, mais partagent aussi des caractéristiques architecturales avec les nos 27, 29, 18 et 20 de la même rue. De sorte qu'on est amené à supposer l'intervention d'un seul et même architecte sur les neuf unités fonctionnelles (repérables chacune par une porte d'entrée sur la rue) ainsi constituées. Cependant, on peut remarquer deux anomalies dans l'adéquation entre les immeubles et leur conception. Tout d'abord, le $n^{\circ} 25$ est manifestement dessiné par une main différente et il forme une interruption dans la série.

De plus, les nos 29, 18 et 20 ont une longueur de façade beaucoup plus importante, les rapprochant des autres immeubles du quartier, à la différence des nos 15 à 23. 
Figure 9

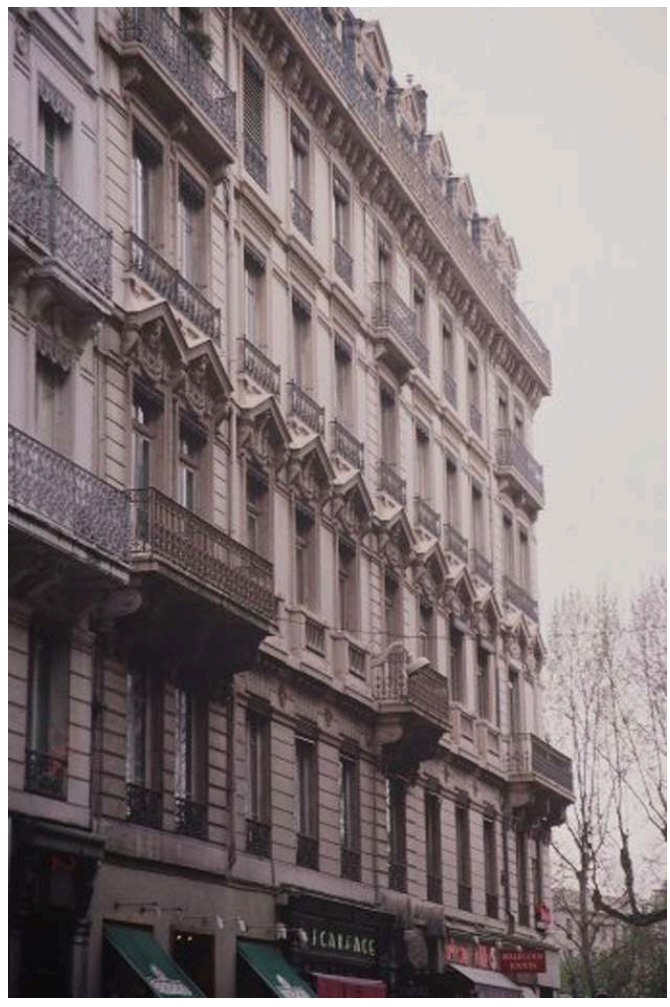

Immeuble $n^{\circ} 29$, rue Gasparin, façade

Phot. B. Gauthiez. (c) B. Gauthiez. 2000

Figure 10

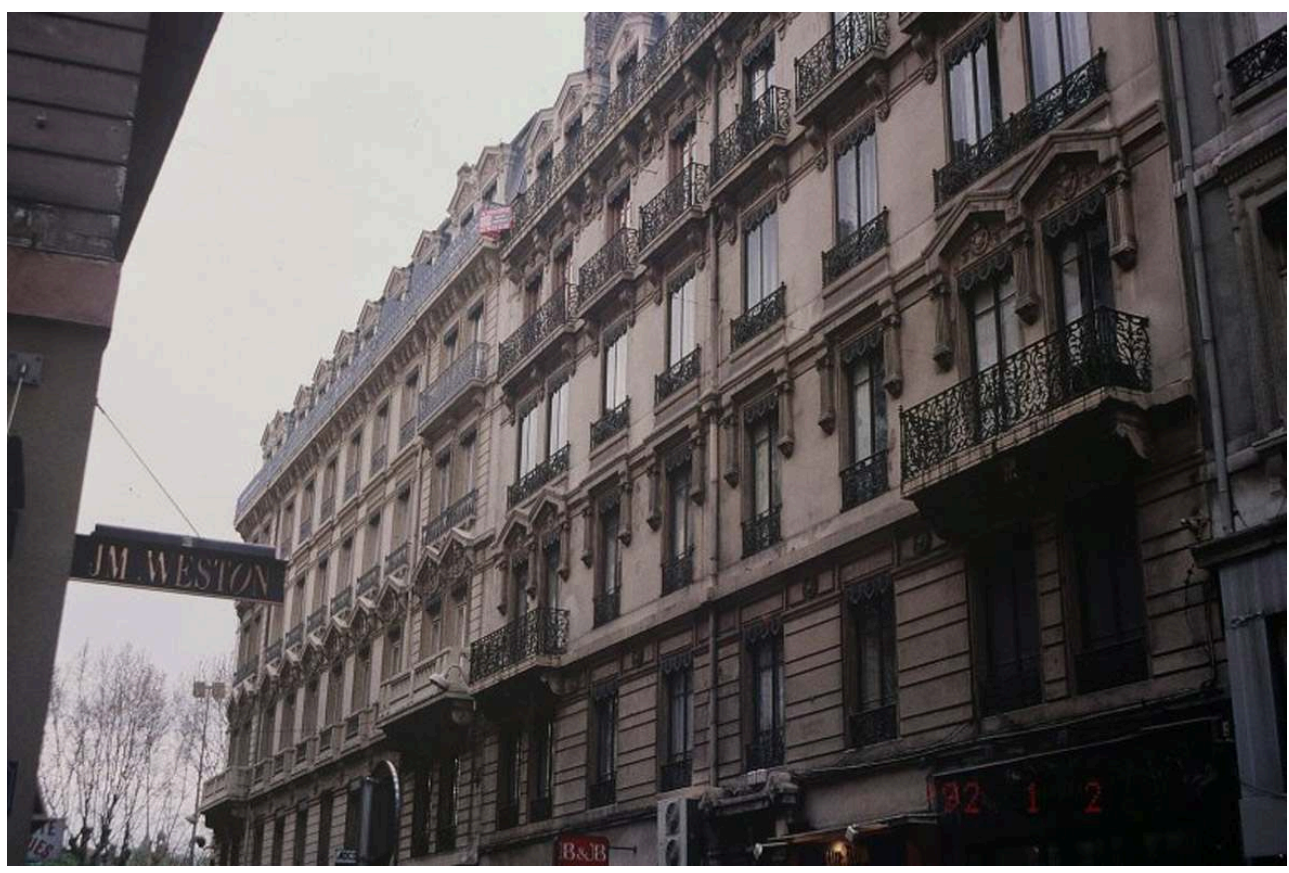

Immeubles $n^{\circ} 18$ et 20 , rue Gasparin, façades

Phot. B. Gauthiez. (c) B. Gauthiez. 2000 
Figure 11

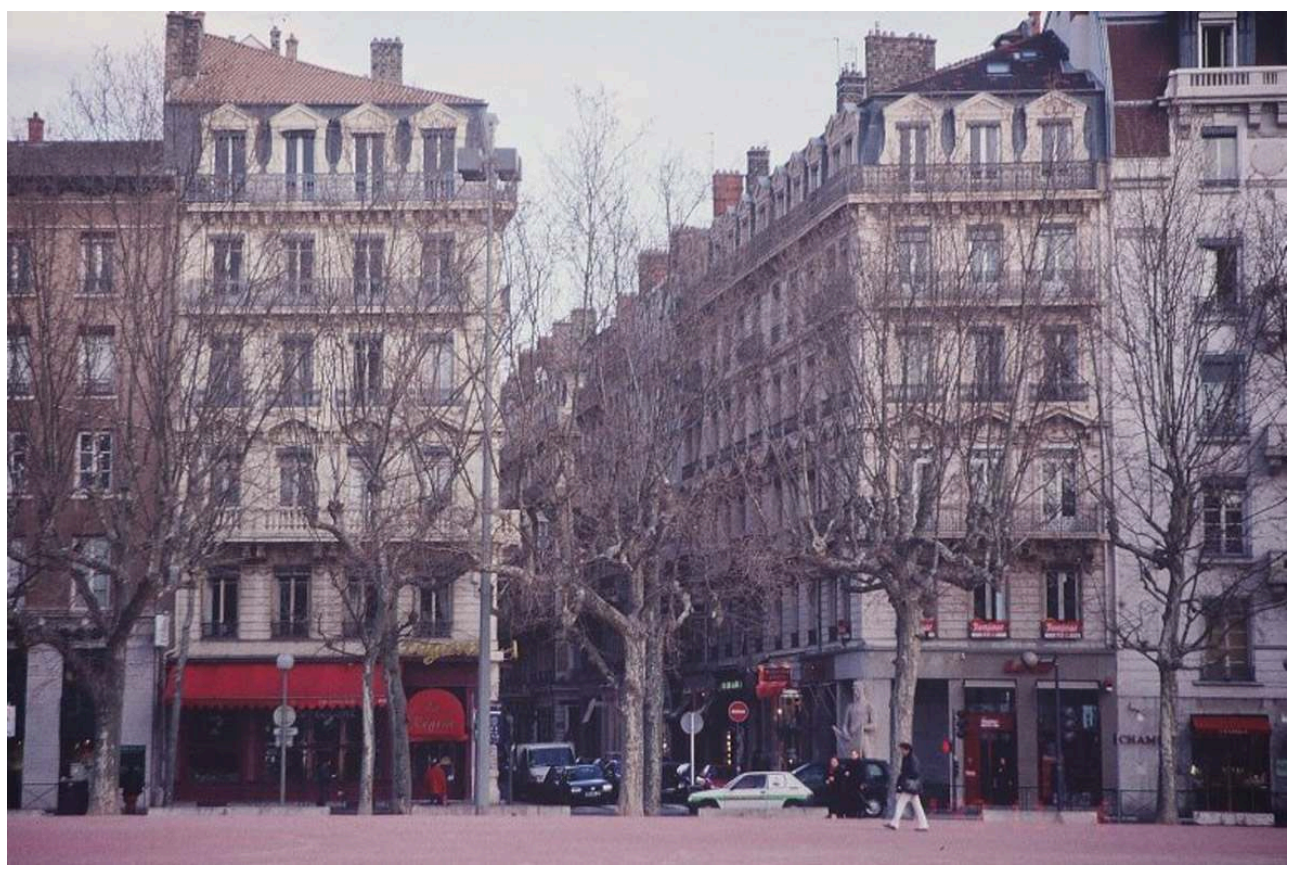

$N^{\circ} 20$ et 29 rue Gasparin, façades en retour sur la place Bellecour

Phot. B. Gauthiez. (c) B. Gauthiez. 2000

9 Nous sommes donc en présence de neuf immeubles, définissables dans l'état actuel par autant d'unités fonctionnelles, chacun sur sa parcelle. Ils partagent en première analyse un même concepteur et les nos 15 à 23 , sans qu'on puisse dire s'ils font partie de la même unité de conception, ont de nombreuses caractéristiques qui font penser qu'ils sont dessinés sur un même canevas et ne varient que par le décor. Dans une approche limitée à ces constats, nous serions donc en présence de neuf édifices distincts. L'histoire de leur production conduit pourtant à rejeter cette classification.

\section{L'histoire d'une production complexe}

L'histoire de ces immeubles peut être retracée, non sans que quelques incertitudes ne subsistent, au travers des archives de la ville et du service des impôts fonciers. Elle peut être décomposée en six étapes principales :

A. En janvier 1860, dans le cadre du percement de la rue de l'Impératrice, l'actuelle rue du Président-Edouard-Herriot, le clos de l'ancienne préfecture est vendu par la ville. Du moins, il est cédé par lots, la vente ayant pu intervenir plus tard. Les immeubles sont localisés dans ce lotissement.

B. L'architecte Etienne Journoud élabore en 1861 un projet de débouché de la rue Gasparin sur la place Bellecour, au sud, en forme de passage couvert. Il concerne les deux côtés de la rue Gasparin qui, parallèle à la rue de l'Impératrice, complète le lotissement du clos de l'ancienne préfecture. Journoud s'associe à l'entrepreneur Pierre Dumont et à un nommé Touzot pour acheter à la ville deux lots de terrains en bordure de la voie nouvelle. L'un, acheté le 19 mars 1862, concerne précisément l'emplacement du projet de 1861, de part et d'autre de la rue. L'autre, acquis le 18 août 1862, concerne un lot situé plus au nord, celui d'un nommé Laville, où seront édifiés plus tard les nos 
15 à 21. Les lots ainsi acquis ont été délaissés par leurs acquéreurs primitifs, parmi lesquels Amédée Savoye, architecte actif dans l'investissement immobilier.

C. Journoud, Dumont et Touzot revendent le lot du futur $\mathrm{n}^{\circ} 15$ à Romain Pilet, menuisier, et Romain Miniot, négociant, puis, à la même date du 25 septembre 1862, celui du $n^{\circ} 25$ à l'entrepreneur Antoine Chavepeyre et à Pierre Falque, le lot du $n^{\circ} 27$ au serrurier Guillaume Lapoire et au notaire François Berloty, enfin celui $d u n^{\circ} 18$ au plâtrier Joseph Gilles et au rentier Charles Barrard. Dumont se lance alors avec Antoinette Colombier dans la construction du $n^{\circ} 21$. Les trois associés entreprennent au même moment celle du ${ }^{\circ} 20$.

D. Les nos 15 et 21 sont achevés en 1863, puis en 1864 les nos 18 et 20 . Le $n^{\circ} 25$ est alors aussi pratiquement terminé. Le $n^{\circ} 15$ est revendu dès le 7 septembre 1863 par Pilet et Miniot. On est alors en plein « boom » des constructions liées au percement de la rue de l'Impératrice.

E. Ce «boom » est suivi d'une crise fortement ressentie, puis d'un redémarrage de la construction. Celle-ci est favorisée par la revente du n ${ }^{\circ} 21$ par Dumont et Colombier le 24 août 1866. Les trois associés initiaux, disposant vraisemblablement alors de fonds suffisants, grossis par les reventes des constructions achevées, entreprennent l'édification des nos 17, 19, 23 et 29 en 1867. Ceux-ci sont terminés en 1867. Le $n^{\circ} 25$ l'est aussi la même année.

F. Une nouvelle crise intervient en 1869, suivie de la revente des immeubles élevés par les trois associés : le $n^{\circ} 23$ le 26 avril 1869, le $n^{\circ} 19$ le 27 janvier 1870 , le $n^{\circ} 20$ deux jours plus tard au notaire Berloty. Le $n^{\circ} 29$ sera revendu seulement neuf ans après sa construction, le 25 août 1876 . Il semble que le $n^{\circ} 17$ soit resté la propriété de Journoud. Le lot du $\mathrm{n}^{\circ} 27$ est vendu en 1870, son édification sera achevée l'année suivante.

11 L'histoire vue par le biais des acteurs et des dates d'achèvement est cependant incomplète, et ne rend qu'en partie compte de la complexité de l'opération. Les trois associés initiaux, Journoud, Dumont et Touzot, ont tracé onze lots. La conception architecturale de 10 lots sera confiée à Journoud, nous le savons par les textes et les signatures portées sur les immeubles. Mais le traitement apporté aux nos 15 à 23 est, on l'a vu, différent de celui des autres. L'entrepreneur Dumont intervient probablement sur six lots, sous la maîtrise d'œuvre de Journoud. Le métier des acquéreurs des nos 15, 18, 25 et 27 doit attirer notre attention: un menuisier, un autre entrepreneur, un serrurier, un plâtrier, autant de corps de métier qui ont pu intervenir, de façon croisée, sur les autres chantiers de l'opération. On est donc amené à supposer une forme d'association dans le montage d'ensemble, association à laquelle on est tenté de rattacher le notaire Berloty, qui a pu jouer un rôle de banquier, comme c'est souvent le cas pour les notaires de cette époque impliqués dans les opérations de rénovation urbaine. Cette association élargie reste, faute de recherches plus larges, du domaine de la conjecture. On sait cependant que Journoud était attentif au sort de ses entrepreneurs. Dans une lettre du 5 avril 1864, en pleine crise, et dans l'incapacité de louer son immeuble du $\mathrm{n}^{\circ} 25$ pourtant pratiquement achevé, l'entrepreneur Chavepeyre exprime son désespoir au préfet-maire de la ville, Vaïsse, parlant des «circonstances fâcheuses qui s'attachent aux entreprises de construction, et principalement à celles de la rue Gasparin, dont les espérances ont été si cruellement déçues ». A la suite de quoi Journoud rachète l'immeuble à Chavepeyre, puis lui donne une chance de se remettre au $\mathrm{n}^{\circ} 27$, avec le nommé Falque. Ces circonstances expliquent probablement que Journoud ne soit pas l'architecte de ce dernier immeuble. 
12 L'aspect foncier de l'affaire n'est pas moins complexe. Les trois associés ont constitué des lots dont ils concèdent une partie à divers investisseurs. Cependant, c'est à eux seuls que la ville a opéré la cession initiale, relative au premier lotissement. De sorte qu'ils sont amenés à lui demander d'accepter que le paiement des lots soit fait directement par les acquéreurs des "sous-lots ", qu'ils ont constitués par le partage des deux grands lots initiaux. Il s'agit d'un nouveau lotissement de fait, permettant aux investisseurs de gager les emprunts nécessaires à la construction sur un bien foncier identifié, ce que Journoud décrit ainsi au maire Vaïsse le 8 mai 1862, s'exprimant au nom des trois associés : «nous ne pouvons, non plus que nos acquéreurs, mener à fin une entreprise de cette importance avec nos seules ressources et sans faire appel à des bailleurs de fonds : chacun des constructeurs devra donc ouvrir un emprunt particulier sur son lot par l'entremise du notaire qu'il aura choisi...». La fragilité des financements, alors que les revenus des constructions sont sujets aux aléas du marché immobilier, est à l'origine de la détresse de l'entrepreneur Chavepeyre, déjà évoquée. Il est intéressant de noter que Journoud ne paiera que très tard, le 22 janvier 1871, le terrain du $n^{\circ} 17$, qu'il s'était réservé. Dans l'ensemble, les terrains des trois associés n'ont probablement été payés à la ville qu'après la vente des immeubles, une fois ceuxci achevés. Il y a donc eu une différence de traitement de la part de la ville entre les investisseurs, avec plus d'indulgence pour ceux qui courraient un risque plus grand.

13 A ce stade de l'enquête, le montage de l'opération permet d'expliquer et de comprendre deux des caractéristiques principales des immeubles des nos 15-23. La taille identique des lots correspondants vient d'un lotissement secondaire opéré par les trois associés à l'origine de l'opération. La petite taille des lots et donc des immeubles, alors que leur hauteur est déterminée par un règlement municipal s'étendant à tout le nouveau quartier, est due à la modestie des investissements à la portée des acquéreurs, de petits entrepreneurs.

\section{L'architecture des immeubles}

Ces éléments ne nous permettent pas, cependant, d'expliquer les caractéristiques architecturales des constructions. Pour ce faire, il est nécessaire d'étendre l'enquête à la carrière d'Etienne Journoud. Celle-ci nous apportera d'autres éclairages sur ses liens avec les entrepreneurs, et donc sur les deux caractères morphologiques précédemment décrits.

Né en 1828, Journoud devient architecte vers 1850, puis travaille dans l'agence de Dupasquier, architecte de renom à Lyon. Il est ensuite engagé par l'architecteinvestisseur Benoît Poncet sur l'immense chantier de la rue Impériale, l'actuelle rue de la République. Poncet y est chargé par la société concessionnaire - la Société anonyme de la rue Impériale, dont il est le principal initiateur - de la maitrise d'œuvre des opérations. Alors que seule la réalisation de la rue était prévue, Poncet convainc la société de procéder à la construction des immeubles qui doivent l'accompagner. Ce faisant, il est chargé de la conception et du suivi du chantier. Il engage dans ce but une équipe de très jeunes architectes, qui paraissent s'être spécialisés dans tel ou tel aspect de la profession. Etienne Journoud, quant à lui, se fait vite remarquer par sa grande énergie et son " courage d'aller disputer tous les matins sur les échafauds » : c'est là à n'en pas douter l'origine de ses relations étroites avec le monde des entrepreneurs. 
Dans l'architecture des immeubles de la rue Impériale, Poncet se trouve confronté à une vive revendication de la Société académique d'architecture de Lyon, qui regroupe les architectes de la ville et des environs, pour qu'il introduise de la variété architecturale dans le paysage de la rue. La revendication a pour base une visée corporatiste: apporter de la variété, c'est faire travailler un plus grand nombre d'architectes et répartir la tâche en ne la laissant pas à une seule agence. C'est aussi une rupture dans la façon de concevoir le rapport de l'individualité architecturale des immeubles (entendus comme unités fonctionnelles) avec l'espace de la rue ou de la place. En effet, jusqu'ici, à Lyon, et lorsque c'était possible, on recherchait plutôt la répétition d'un unique motif, même sur de grandes longueurs, à l'exemple de la rue de Rivoli à Paris ou du quai de Rouen, réalisations de la première moitié du siècle. Pour répondre à cette revendication, Poncet confie la conception des façades à ses jeunes architectes, d'où une diversité évidente encore aujourd'hui dans le paysage de la rue. Cette diversité est introduite par une répartition en groupes d'immeubles, au sein desquels les façades sont distinguées par de légères variations de décor. Or, c'est précisément de la sorte que Journoud a conçu les nos 15-23 rue Gasparin. Si les caractéristiques d'ensemble sont différentes de celles de la rue Impériale, on y retrouve des plans types, des hauteurs de niveau identiques entre les immeubles, des solutions constructives répétitives, même si l'on a construit à des dates différentes. Enfin, les variations reproduisent dans le décor du détail celles qu'on peut constater rue Impériale, avec un peu plus de richesse décorative, phénomène partout présent dans le percement de la rue de l'Impératrice et ses abords. Poncet a donc fait ici école, du point de vue de l'architecture comme d'ailleurs de celui de l'investissement, puisqu'il s'efforçait « constamment de faciliter à ses employés les occasions de spéculation qui pouvaient se présenter dans les nombreuses entreprises qu'il a faites ».

\section{Conclusion}

17 On voit combien il est, dans le cas de ces immeubles de la rue Gasparin, difficile de trancher entre une monographie portant sur un immeuble unique et une monographie portant sur le groupe, lui-même fruit d'un système de production plus large. L'unité de fonds, qu'on la constate à l'origine de l'opération ou après son achèvement, n'est pas ici une approche pertinente. L'unité de fonction-destination, mettant l'accent sur l'émiettement des immeubles, ne l'est pas non plus. Il n'y a pas de correspondance entre fonds et fonction, si ce n'est une fois l'opération terminée. C'est, dans ce cas, l'unité de conception qui doit guider la démarche, puisqu'elle est la seule à nous permettre de remonter le fil de l'opération et, surtout, de comprendre la forme effective des immeubles devant lesquels nous nous trouvons. C'est d'autant plus vrai que ce type de situation, qui voit deux immeubles ou plus - définis ici comme unités fonctionnelles - produits selon une même conception architecturale mais individualisés par leur décor secondaire, est très fréquent à Lyon depuis l'opération de la rue Impériale jusqu'au début du $\mathrm{XX}^{\mathrm{e}}$ siècle.

18 En ce sens, notre groupe d'immeubles est représentatif d'une famille d'immeubles multiples. Cependant, l'opération dans laquelle il s'insère en inclut d'autres avec lesquels il partage, à l'exception d'un seul, un même concepteur. L'opération elle-même est remarquable par son montage et ses produits architecturaux. Il faut donc s'intéresser à la fois au lotissement de l'ancien clos de la Préfecture, au lotissement des 
trois associés Journoud-Dumont-Touzot avec ses conséquences architecturales, et aux immeubles qui ont été produits dans ce cadre. Chacun de ces objets doit être étudié.

BIBLIOGRAPHIE

\section{Archives départementales du Rhône}

3 P 123 - 30 et 3 P 123 - 33, matrice cadastrale et mutations 1837-1913, secteur Bellecour.

\section{Archives municipales de Lyon}

321 WP 56, dossier rue Gasparin.

321 WP 123, dossier rue de l'Impératrice.

321 WP 175, dossier rue Impériale.

\section{Bibliographie}

- Carnutensis, « Nécrologie, Etienne Journoud », La construction lyonnaise, 1er janvier 1897, 19e année, $n^{\circ} 1$.

- Gauthiez Bernard, Lyon entre Bellecour et Terreaux, architecture et urbanisme au XIX ${ }^{e}$ siècle, Editions lyonnaises d'art et d'histoire, Lyon, 1999.

- Tisseur Clair, « Benoît Poncet et sa part dans les grands travaux publics de Lyon », Bulletin de la société académique d'architecture de Lyon, T. VII, 1881-2, p. 105-188.

INDEX

Mots-clés : inventaire général, en ligne, journal, revue électronique, revue numérique, périodique, patrimoine, histoire de l'art, France, architecture, urbanisme, agglomération, RhôneAlpes

Keywords : on line, electronic journal, ejournal, heritage, history of art, France, architecture, settlements, Rhône-Alpes

\section{AUTEUR}

\section{BERNARD GAUTHIEZ}

Architecte urbaniste en chef de l'Etat, Sous-direction des études, de la documentation et de l'Inventaire, Hôtel de Vigny 10, rue du Parc-Royal 75003 Paris. bernard.gauthiez@culture.gouv.fr 